\title{
Jenseits der Zulassungsstudien
}

Ergebnisse aus Zulassungsstudien sowie Leitlinienempfehlungen können eine Entscheidungshilfe bei der Auswahl eines Antiepileptikums (AED) sein. Darüber hinaus nehmen Präparateigenschaften und das Interaktionspotenzial Einfluss auf die Entscheidung. Welches AED sich besonders für die Kombinationstherapie eignet, diskutierte eine Expertenrunde.

Ihre Forderungen: Außer auf die Wirksamkeit und Verträglichkeit des AED sollte darauf geachtet werden, dass es rasch und einfach aufzudosieren ist. Vor dem Hintergrund der Mehrfachmedikation aufgrund von Komorbiditäten sollten AED mit fehlendem oder geringem Enzyminduktions- beziehungsweise Interaktionspotenzial zum Einsatz kommen, empfahl Professor Christoph Baumgartner, Wien. Dazu zählen unter anderem Lacosamid und Levetiracetam.
Sehr viele Epilepsie-Patienten benötigen im Krankheitsverlauf eine AED-Mehrfachkombination, so Dr. Stefan Stodieck, Hamburg. Hier gilt es, die „Drug load“ möglichst gering zu halten. Dies gelingt, indem bei einer Add-on-Therapie, etwa mit Lacosamid, die Dosis der Basismedikation reduziert wird.

Für Lacosamid liegen seit vier Jahren Praxiserfahrungen vor. Gute Wirksamkeit und Verträglichkeit, ein unkompliziertes, schnelles Auftitrieren, ein rascher Wirkungseintritt sowie geringes Interaktionsrisiko kennzeichnen das Antiepileptikum. Lacosamid steht nicht nur in Form von Filmtabletten zur Verfügung, sondern auch als Sirup und Infusionslösung. Dr. Carin Szostecki, Springer Medizin

UCB-Symposium bei der 8. Ligatagung Epilepsie, Interlaken, 9.5.2013; Veranstalter: UCB Pharma

\section{Früh mit der Therapie beginnen und im Dialog bleiben}

Die Diagnose MS kann heute bereits nach dem ersten klinischen Schub gestellt werden. „Der Zeitgewinn durch die frühere Diagnosestellung kann therapeutisch genutzt werden, um eine Akkumulation von Behinderungen zu vermeiden“, berichtete Professor Michael Sailer, Median Klinik NRZ Magdeburg. Bereits im Frühstadium sind entzündliche Prozesse mit irreversiblen axonalen und neuronalen Schädigungen assoziiert und die Schubzahl zu Krankheitsbeginn korreliert mit einer beschleunigten Behinderungsprogression. Das sei auch die Rationale für einen frühzeitigen Therapiebeginn mit einer stark wirksamen Dosierung von Interferon beta (IFN $\beta$-1a), erläuterte Professor Friedemann Paul von der Charitè Berlin. Der Nutzen einer Frühtherapie zeigte sich auch in der IMPROVE-Studie [De Stefano N et al. Mult Scler 2010; 16: 888-92]: Bei Patienten mit schubförmig remittie- render MS, die mit subkutanem IFN $\beta$-1a (Rebif ${ }^{\circledR} 44 \mu \mathrm{g} ; 3 \mathrm{x}$ wöchentlich) behandelt wurden, war die durchschnittliche Anzahl kombinierter einzelner aktiver Gehirnläsionen nach 16 Wochen um $69 \%$ reduziert $(p<0,001)$. Neben einer frühen Diagnose der MS und einer rasch wirkenden immunmodulatorischen Basistherapie können ein vertrauensvoller Arzt-Patienten-Kontakt und die Unterstützung durch MS-Schwestern zu einer dauerhaften Therapieadhärenz beitragen. Dazu gehört auch die Verwendung des patientenfreundlichen elektronischen Injektors $\left(\right.$ RebiSmart $\left.^{\mathrm{Tu}}\right)$ in Verbindung mit einem Betreuungsprogramm wie dem zertifizierten RebiSTAR ${ }^{\mathrm{T} \omega}$-Programm.

Abdol A. Ameri, freier Medizinjournalist

Meet-the-Clinic „Mehr Dialog für mehr Adhärenz: Wie MS-Patienten länger im grünen Bereich bleiben“ Berlin, 11.6.2013; Veranstalter: Merck-Serono

\section{Erstes Tranylcypromin- Generikum}

Ab sofort ist Tranylcypromin-neuraxpharm ${ }^{\oplus}$ (in den Wirkstärken $10 \mathrm{mg}$ und $20 \mathrm{mg}$ ) zur Behandlung von depressiven Syndromen unabhängig von ihrer nosologischen Einordnung erhältlich. Die viertelbare 20 -mg-Filmtablette ermöglicht es, im gesamten Therapieverlauf nur eine Tablettenform einzusetzen.

Nach Informationen von neuraxpharm

\section{Zwei Meilensteine der MS-Therapie erreicht}

Der Arzneimittelausschuss (CHMP) der Europäischen Arzneimittelagentur (EMA) hat eine positive Stellungnahme zur Zulassung von Alemtuzumab (Lemtrada ${ }^{\circledR}$ ) für die Behandlung von Erwachsenen mit schubförmig-remittierender Multipler Sklerose bei Nachweis von Krankheitsaktivität im klinischen Befund oder in der Bildgebung abgegeben. Außerdem empfahl das CHMP, Teriflunomid (Aubagio ${ }^{\circledR}$ ) den Status eines neuen Wirkstoffs (new active substance, NAS) zu erteilen. Im März dieses Jahres hatte sich das CHMP bereits positiv zur Zulassung von Teriflunomid zur Therapie Erwachsener mit schubförmig-remittierender MS geäußert.

Nach Informationen von Genzyme - A Sanofi Company

\section{Alfred-Hauptmann-Preis}

Im Rahmen der 8. Dreiländertagung der Deutschen und Österreichischen Gesellschaft für Epileptologie und der Schweizerischen Liga gegen Epilepsie erfolgte die feierliche Übergabe des Alfred-Hauptmann-Preises an Professor Yvonne Weber aus Tübingen für ihre Arbeit zu PRRT2-Mutationen als Hauptursache benigner familiärer infantiler Krampfanfälle. Die Arbeit leistet einen Beitrag zum besseren Verständnis der Pathophysiologie von genetisch bedingten Epilepsien. Der seit 2009 von dem Unternehmen UCB geförderte Preis ist mit $10.000 €$ dotiert.

Nach Informationen von UCB 